\title{
LIDAR technique application to the analysis of environmental pollution
}

\author{
S. Torno, J. Toraño, M. Menendez, M. Gent \& C. Allende \\ School of Mines, University of Oviedo, Spain
}

\begin{abstract}
Landfill sites are the simplest and most common ways to eliminate urban solid waste, although large amounts of pollutants are introduced into the environment, such us dust, gas, soluble and insoluble substances.

Both the digital model of the landfill surface and the three-dimensional meshing of the domain, where the dispersion phenomena is analyzed, are essential when modelling pollutant behaviour using Computational Fluid Dynamics (CFD3D).

The commonly used Digital Surface Models are generated by combining a set of geometric primitives. These are obtained through a process of scanning, in which a set of points, which geometrically defines the landfill site, are identified by the derived cartography.

In this study, we propose a procedure to digitally model a real surface from a data point cloud obtained through LIDAR (Light Detection and Ranging) technology, which identifies, in real-time, the main geometry that defines its surface. Thus, these landfill digital models are exported into the Ansys CFX 10.0 software for numerical modelling, in order to analyze the behaviour of dust and odours generated by the landfill site.

The importance of LIDAR data technology in the study of environmental pollution produced by landfill sites is supported by the correlation between the obtained results through modelling and those obtained through field measurement programmes.
\end{abstract}

Keywords: LIDAR (Light Detection and Ranging), CFD3D (Computational Fluid Dynamics), environmental pollution, landfill site. 


\section{Introduction}

Environmental pollution has become a serious challenge for humans and a greater concern for scientist and researchers worldwide. The increase of both industrial development and urban population growth has produced an increase of solid and liquid waste, which leads to environmental degradation, mainly through processes of dispersion of these pollutants.

Landfill sites have become an important problem for our society as they have a limited lifetime. This problem is even more accentuated in developing countries, since population growth leads to an increase in the amount of waste generated, exhausting the landfill site capacity.

Literature suggests that adverse sensory reactions to strong odours and irritants, may lead to health problems. With regard to dust pollution, it was commonly thought that the effect of these particles on human health was limited to the respiratory system [1], but recent studies have demonstrated both its carcinogenic action and contribution to cardiovascular diseases.

Atmospheric pollution studies and analyses based on computational models are innovative techniques that can predict the behaviour of the various air pollution processes due to dust particles and odour dispersion. The generation of this type of model consists of three phases: definition of the landfill geometry, three-dimensional meshing of the computational domain, and resolution of the model.

Currently, the methodology used when designing geometrically a landfill site is based on the extraction of information from a three-dimensional data set obtained from a scanning process by cartography and the subsequent model drawing using SolidWorks software. The origin of this cartographic information is generally a photogrammetric process. This model design involves a set of processes: Planning and implementation of a photogrammetric flight in which a set of aerial photographs of the studied area are obtained.

By means of a Photogrammetric Restitution process, a three-dimensional scale model of the area is obtained, where both the identification of the most important elements and three-dimensional coordinates of the mentioned area are determined. A drawing process of these elements which create cartography to a specific scale has been used.

Selection and extraction of three-dimensional information of the landfill site have been carried out by an interpolation process, which implies an important adjustment for the accuracy of the set of points which is required for the model design. These set of points constitute the landfill model which has been applied to the environmental pollution study.

Data acquisition LIDAR (Light Detection and Ranging) technology has been used for the generation of the landfill geometry. This technology provides a reliable and accurate, but irregular representation of the terrestrial scenarios from a 3D-coordinate $(\mathrm{X}, \mathrm{Y}, \mathrm{Z})$ point cloud [2] using a sensor installed on an airborne platform. Being an irregular representation, it requires data processing so as to obtain a regular and simplified landfill site model. 
This technology shows important advantages when considering the latter processes reducing labour and increasing the accuracy by directly obtaining three-dimensional information, from the same flight from where aerial photographs have been obtained. On the other hand, the need to select and interpolate the set of points for the design of the model was replaced by automated techniques for the identification and adjustment implicit in the processing of the point cloud.

Several implicit processes to handle this information and literature related to different automatic algorithms have been used. These algorithms can be classified into: filtering algorithms, classification algorithms and segmentation algorithms.

Firstly, the original point cloud data are submitted to a filtering process [3] in order to identify points which belong to the surface. This process can be carried out according to different criteria, which can be classified as mathematical morphology-based filters and directional ground filters, [4]. The morphologybased filters are local neighbourhood systems, [5], while directional filters apply different analysis criteria and are of global type: Robust Interpolation [6], technique active contours model [7], contour clustering [8], multi-directional ground filtering [4] or based on Occlusion Detection [9].

Once the landfill point cloud has been filtered, a LIDAR data set that belongs to the bare ground is obtained and ready to be processed. This process is called segmentation and consists of identifying geometric primitive sets. There are several algorithms that perform this process automatically, differentiating between linear primitives [10] and planar primitives [11].

The equations governing fluid flow problems (related mostly to the effect of wind in our case) that produce entrainment (dispersion) of pollutants on their way through landfill sites are the Navier-Stokes, the continuity and the energy equations. These equations, despite being linear, only allow analytical solutions for simple geometries, otherwise Computational Fluid Dynamics (CFD) are used.

The direct resolution of the Navier-Stokes, or the DNS ('Direct Numerical Simulation') equations is the most obvious and accurate way to predict turbulent flow. Nevertheless, from all the Eddy-viscosity models, based on RANS (Reynolds Average Navier Stokes) equations, we have chosen the Standard Model K-epsilon turbulence, as it is the most widely used in engineering, providing the best results when solving fluid flow in scenarios like the one studied, and its adjustment through range survey $[12,13]$.

\section{Landfill site features}

A landfill site is an engineering work whose final purpose is the disposal of solid wastes, which are laid on the ground under controlled conditions which minimize its adverse effects on the environment and human health.

Nowadays, sanitary landfills are constructed to preserve existing landfills. These places are used to store waste materials that are compacted to reduce their volume, by alternating $40 \mathrm{~cm}$ layers of soil and other materials. Multiple 
measures are taken to reduce the problems generated by other treatment methods. These measures include the study of environmental, economic and social impact, planning and choosing the location, as well as monitoring and analyzing the site during its lifetime.

Their morphology is the combination of planar-fittings with a regular slope, and berms (width of strips between lifts) arranged in a pyramidal shape, and bounded by sloped lines or break lines. As for artificial slopes, they can be considered almost homogeneous. This facilitates the processing of the original point cloud data set obtaining a simplified real digital model, using simple segmentation algorithms.

The studied landfill site is located in the municipal district of Cudillero close to the Northern coast of Asturias (Spain), and nearby to the Cantabrian Motorway, as shown in figure 1.

Figure 2 shows the 3D LIDAR data point cloud from which the irregular terrain geometry is extracted.
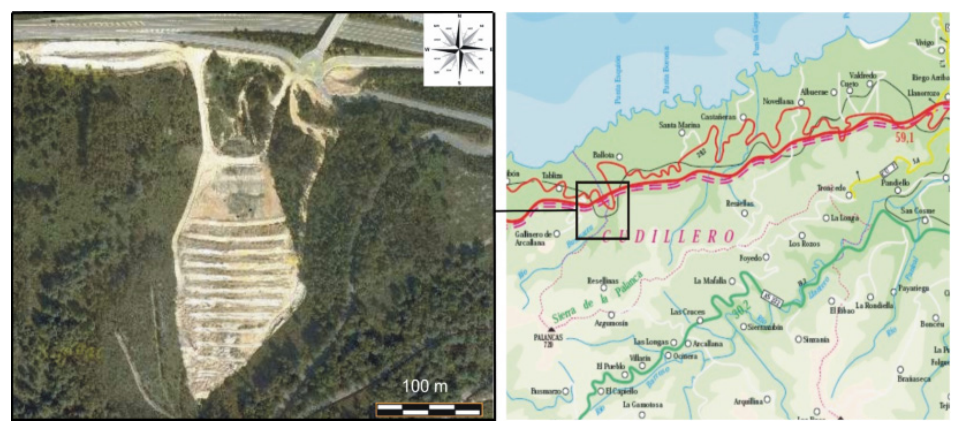

Figure 1: Landfill location. Asturias (Spain).

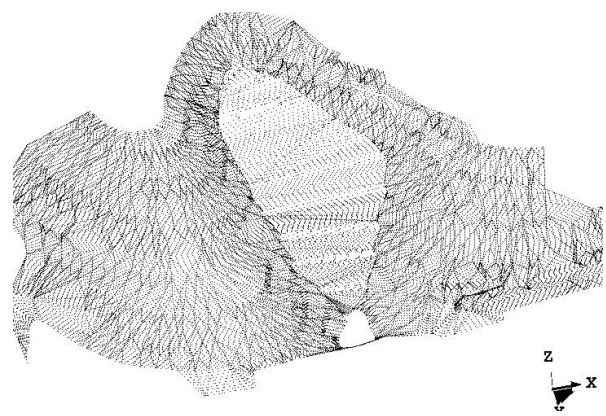

Figure 2: $\quad 3 \mathrm{D}$ data set point cloud. 

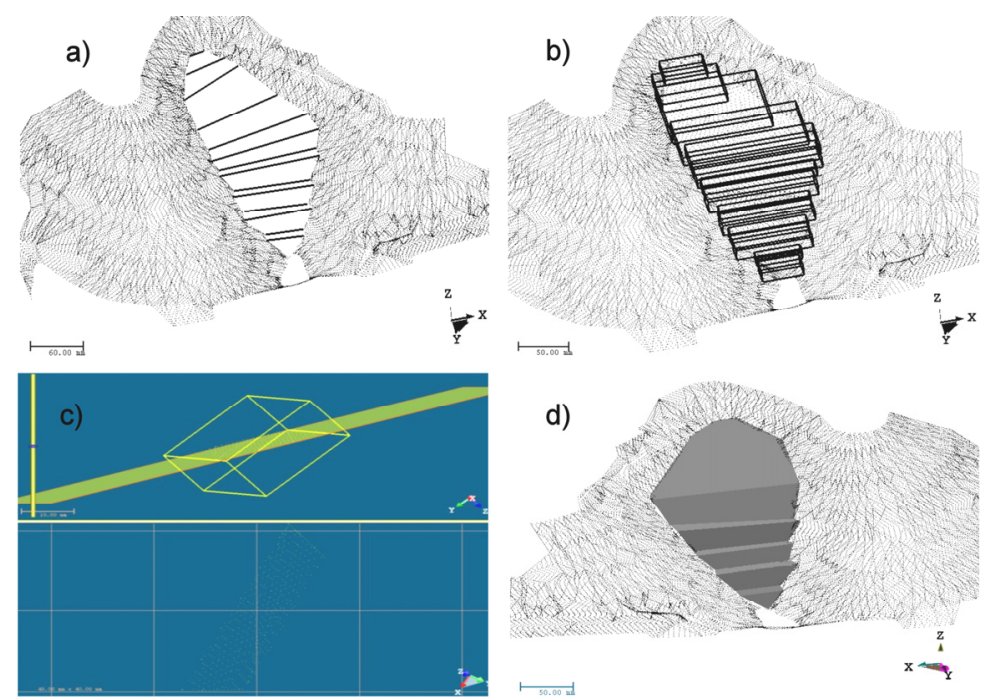

Figure 3: a) Linear segmentation. b) Set of points to segment in plane surfaces. c) Statistical determination of plane geometries. d) Simplified geometry of the landfill.

\section{Processing data set}

For the pollutant emission simulation, by Computational Fluid Dynamics, the generation of a digital model, as a simplified representation of the terrain that preserves some of its properties, is necessary. Its main objective is the study of the emission in a simple but understandable manner. That is, the original object is represented by another object of a lesser complexity in order to predict its properties.

The linear primitives from the point cloud delimiting: a) the landfill planarfitting and berms geometry, b) the landfill boundaries with natural terrain are identified. This data processing is carried out by the RealWorks Trimble Survey Office Advanced software (figure 3a).

Data processing is directly carried out from the original point cloud to avoid systematic errors due to interpolation, which is a necessary step to generate the digital model. Once break lines are identified, they help us to limit point sets, which belong to each level, so as to perform the segmentation process (figure 3b).

The process of segmentation of plane surfaces is conducted using a least squares fitting to obtain the plane with the least distance to each point (figure $3 \mathrm{c})$.

When the distance from each measured point to the planar geometry is greater than a predetermined limit or threshold, subsets of points and smaller planar geometries, better suited for the real geometry of the model, are defined. This generates geometric primitives adapted to the terrain depending on accuracy 
requirements. In this project, plane surfaces have generated a standard deviation of $0.10 \mathrm{~m}$, accurate enough for a reliable estimation of the computer simulation.

Finally, this process is applied to all point sets to determine the simplified geometry model of the landfill geometry (figure $3 \mathrm{~d}$ ).

\section{Computational simulations}

The simplified geometry of the landfill site surface is used to create the model mesh which is to be simulated. In this research, model meshing is carried out through IcemCFD 10.0 software and is made up of 764,364 of 0.4 tetras.

The mesh data is exported to Ansys CFX-10.0 software where simulation is physically defined. This software is based on Computational Fluid Dynamics (CFD) in order to simulate the wind flow in the model. CFD calculates by resolving a series of partial differential equations [14]. Fundamentally, these equations are Navier-Stokes, eqn (1), Continuity, eqn (2) and Energy eqn (3).

$$
\begin{gathered}
\rho \frac{\mathrm{D} \overline{\mathrm{V}}}{\mathrm{Dt}}=-\bar{\nabla} \mathrm{p}+\rho \overline{\mathrm{g}}+\mu \nabla^{2} \overline{\mathrm{V}} \\
\frac{\mathrm{D} \rho}{\mathrm{Dt}}+\rho \bar{\nabla} \cdot \overline{\mathrm{V}}=0 \\
\rho \frac{\mathrm{D} \tilde{\mathrm{u}}}{\mathrm{Dt}}=\mathrm{K} \nabla^{2} \mathrm{~T}-\mathrm{p} \bar{\nabla} \cdot \overline{\mathrm{V}}
\end{gathered}
$$

where $\rho$ is the density, $\nabla \cdot$ is the operator divergence, $\nabla$ is the gradient, $\mu$ is the viscosity, $T$ is the temperature, $t$ is the time, $V$ is the velocity, $\tilde{u}$ is the specific heat and $K$ is the conductivity.

As has been before mentioned, we have used K-epsilon turbulence model in our simulations of wind effect over the landfill site surface.

Meteorological information obtained from the closest station to the landfill site was introduced into the simulations through two inlets (where wind enters the model) with a 45 degree wind direction to the landfill site longitudinal axis which is characterised by a wind logarithmic profile. This logarithmic profile has a velocity of $1.5 \mathrm{~m} / \mathrm{s}$ at a height of 10 meters over the terrain surface and is implemented into the software through USEPA [15] formula (4) which relates the wind velocity $(\mathrm{u})$, the friction velocity $\left(\mathrm{u}^{*}\right)$, the roughness $\left(\mathrm{z}_{0}\right)$ and height over landfill site surface (z), eqn (4):

$$
\mathrm{u}(\mathrm{z})=\frac{\mathrm{u}^{*}}{0.4} \ln \frac{\mathrm{z}}{\mathrm{z}_{\mathrm{o}}}
$$

where 0.4 is the constant of von Karman.

In figure 4 the boundary conditions simulation, inlet, opening (the air enters and emerges) and wall (all surfaces which act as a wall). 


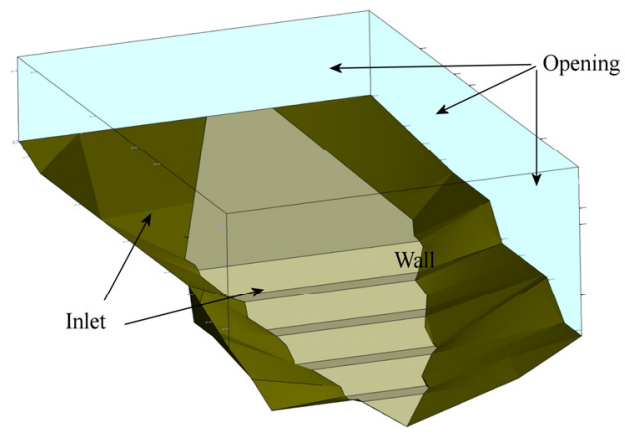

Figure 4: Boundary condition simulation distribution.

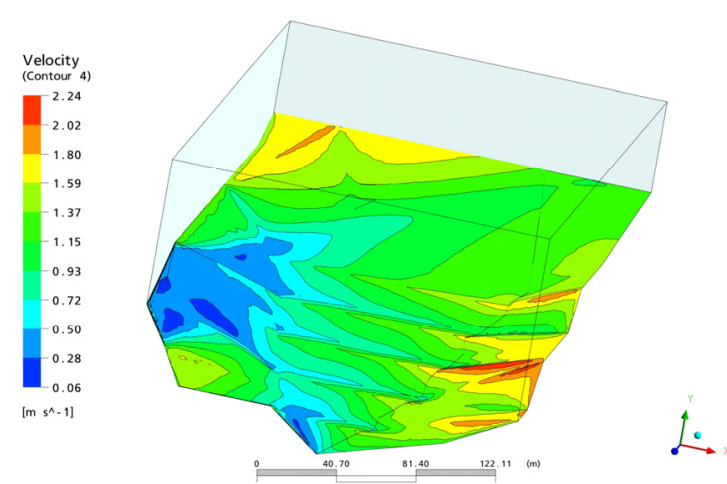

Figure 5: Mean wind velocity distribution in landfill site area (colour online only).

In figure 5 , the mean wind velocity distribution over both the landfill site surface and the surrounding hills is shown. Bright coloured areas (red) correspond to the greater velocity areas whereas the dark coloured areas (blue) correspond to lower or null velocity zones.

Erosion is possible in high velocity value zones (red) whereas a possible material deposit is produced in low velocity zones (blue).

In figure 5 it is observed how the hills create a shade effect over the landfill surface diminishing velocity, which in these areas, leads to a lower particle emission to the atmosphere.

These models need to be validated with real data in order to determine the velocity distribution over the landfill site surface to coincide with real occurrences. Therefore, a series of measurement programmes in the studied landfill site have been carried out. 180 measurement points of the mean wind velocity through a hot wire anemometer (TSI Velocicalc Plus 8386) at a height of $20 \mathrm{~cm}$ from the landfill surface every 5 meters in a straight line through the landfill site centre (landfill longitude being 305 meters) have been carried out. These experimental data are compared to those obtained by the CFD simulations 
(measured at the same points) achieving a 0.94 correlation corresponding to the equation $\mathrm{y}=0.8495 \mathrm{x}+0.2321$.

\section{Conclusions}

In the environmental pollution modelling produced by both organic material and industrial and mining residues landfills the Computational Numerical Codes (CFD3D) is more extensively applied. This software enables us to analyze the wind behaviour crossing the landfill and the effect of this wind dragging the pollutants (dust or gases).

From the 3 modulus in which the CFD software is divided (geometry, meshing, resolution), the application of the LIDAR technique to the real definition of the model geometry, avoiding the use of tools which excessively simplify the model, enables us to achieve a more precise result in pollutant modelling behaviour.

\section{References}

[1] Armstrong, J.A., Russell, P.A., Sparks, L.E. \& Drehmel, D.C., Tethered Balloon Sampling Systems for Monitoring Air Pollution. JAPCA, 31, pp. 735-743, 1980.

[2] Mallet, C. \& Bretar F., Full-waveform topographic LIDAR: State-of-theart. ISPRS Journal of Photogrammetry and Remote Sensing, 64, pp. 1-16, 2009.

[3] Sithole, G. \& Vosselman, G., Experimental comparison of filter algorithms for bare-Earth extraction from airborne laser scanning point clouds. ISPRS Journal of Photogrammetry and Remote Sensing, 59, pp. 85-101, 2004.

[4] Menga, X., Wang, L., Silván-Cárdenas, J.L. \& Currit, N., A multidirectional ground filtering algorithm for airborne LIDAR. ISPRS Journal of Photogrammetry and Remote Sensing, 64, pp. 117-124, 2009.

[5] Filin, S. \& Pfeifer, N., Neighborhood Systems for Airborne Laser Data. ISPRS Journal of Photogrammetric Engineering and Remote Sensing, 71, pp. 743-755, 2005.

[6] Kraus, K. \& Pfeifer, N., Determination of terrain models in wooded areas with airborne laser scanner data. ISPRS Journal of Photogrammetry and Remote Sensing, 53, pp. 193-203, 1998.

[7] Elmqvist, M., Ground surface estimation from airborne laser scanner data using active shape models. The international Archives of Photogrammetry, Remote Sensing and Spatial Information Sciences, 34 (Part 3A), pp. 115118, 2002.

[8] Roggero, M., Airborne laser scanning clustering in raw data. The international Archives of Photogrammetry and Remote Sensing, 34 (Part 3/W4), pp. 227-232, 2001.

[9] Chang, Y-C., Habib, A.F., Leeb, D.C. \& Yom, J-H., Automatic classification of LIDAR data into ground and nonground points. The 
International Archives of the Photogrammetry, Remote Sensing and Spatial Information Sciences, 37 (Part B4), pp. 457-462, 2008.

[10] Kraus, K. \& Pfeifer, N., Advanced DTM generation from LIDAR data. The international Archives of Photogrammetry and Remote Sensing, 34 (Part 3/W4), pp. 23-30, 2001.

[11] Zeng, Q., Data filtering and feature extraction of urban typical objects from airborne LIDAR point cloud. The International Archives of Photogrammetry and Remote Sensing and Spatial Information Sciences, 37 (Part B3b), pp. 153-156, 2008.

[12] Toraño, J., Rodriguez, R., Diego, I., Rivas, J.M. \& Pelegry, A., Influence of the pile shape on wind erosion. CFD emission simulation. Applied Mathematical Modeling, 31 (11), pp. 2487-2502, 2007.

[13] Toraño, J., Torno, S., Menéndez, M., Gent, M., Dust emission calculations in open storage piles. Environmental Fluid Mechanics, 9, pp. 493-513, 2009.

[14] ANSYS CFX-Solver, Release 10.0: Particle Transport Theory, 2008. Lagrangian Tracking Implementation, pp. 174.

[15] United States Environmental Protection Agency (USEPA). Update of Fugitive Dust Emissions Factors In AP-42, 1988. Section 11.2-Wind Erosion, MRI No. 8985-K, Midwest Research Institute, Kansas City, MO. 